\title{
Forecast opportunities for European summer climate ensemble predictions using Self-Organising Maps
}

\author{
Julianna C. Oliveira \\ julianna.carvalho@hzg.de \\ Helmholtz-Zentrum Geesthacht, \\ Institute of Coastal Research \\ Geesthacht, Germany \\ International Max Planck Research \\ School on Earth System Modelling, \\ Max Planck Institute for Meteorology \\ Hamburg, Germany
}

Thomas Ludwig

Deutsches Klimarechenzentrum

Hamburg, Germany

\author{
Eduardo Zorita \\ Helmholtz-Zentrum Geesthacht, \\ Institute of Coastal Research \\ Geesthacht, Germany
}

\author{
Vimal Koul \\ Helmholtz-Zentrum Geesthacht, \\ Institute of Coastal Research \\ Geesthacht, Germany \\ Institute for Oceanography, Center \\ for Earth System Research and \\ Sustainability, Universität Hamburg \\ Hamburg, Germany
}

\author{
Johanna Baehr \\ Institute for Oceanography, Center \\ for Earth System Research and \\ Sustainability, Universität Hamburg \\ Hamburg, Germany
}

\begin{abstract}
Current state-of-the-art dynamical seasonal ensemble prediction systems (EPS) still show limited predictive skill, particularly over Europe in summer. We propose a neural network-based classification of individual ensemble members before performing a hindcast skill analysis. This classification targets high skill cases emerging from large-scale atmospheric regimes associated with the dominant modes of summertime low-frequency variability in the North Atlantic-European sector (NAE). This classification allows to then select those ensemble members that better predict the phase of the summer North Atlantic Oscillation (SNAO) and East Atlantic Pattern (EAP). Our baseline is a set of teleconnection patterns in NAE identified by Self-Organising Maps (SOM) using ERA-20C reanalysis data. We illustrate our methodology with an example with one set of hindcast ensemble simulations with 30-members generated by the MPI Earth System Model. We achieve better predictive skills at 3-4 months lead time for sea level pressure and geopotential height anomalies at $500 \mathrm{hPa}$.
\end{abstract}

\section{CCS CONCEPTS}

- Applied computing $\rightarrow$ Environmental sciences.

\section{KEYWORDS}

ensemble prediction, European climate, self-organising maps

ACM Reference Format:

Julianna C. Oliveira, Eduardo Zorita, Vimal Koul, Thomas Ludwig, and Johanna Baehr. 2020. Forecast opportunities for European summer climate

Permission to make digital or hard copies of part or all of this work for personal or classroom use is granted without fee provided that copies are not made or distributed for profit or commercial advantage and that copies bear this notice and the full citation on the first page. Copyrights for third-party components of this work must be honored.

For all other uses, contact the owner/author(s).

CI2020, September 22-25, 2020, virtual, United Kingdom

(c) 2020 Copyright held by the owner/author(s).

ACM ISBN 978-1-4503-8848-1/20/09.

https://doi.org/10.1145/3429309.3429319 ensemble predictions using Self-Organising Maps. In 10th International Conference on Climate Informatics (CI2020), September 22-25, 2020, virtual, United Kingdom. ACM, New York, NY, USA, 5 pages. https://doi.org/10.1145/ 3429309.3429319

\section{INTRODUCTION}

Leading modes of atmospheric variability relevant for European summer climate are linked to the North Atlantic Jet (NAJ). In the North Atlantic-European sector (NAE), the NAJ modulates the occurrence and persistence of weather regimes and controls the location of storm tracks, thus acting as a key driver for temperature and precipitation extremes $[3,6]$. Nevertheless, current state-of-theart climate models strive in accurately representing NAJ variability and likely consequently show limited predictive skill for summer forecasts, particularly over Europe [2]. We present a neural networkbased framework to circumvent such weak dynamical links in the model and achieve improved summer forecast skill.

Dynamical seasonal predictions are obtained by ensemble of simulations in Ensemble Prediction Systems (EPS), for which predictive skill is usually assessed by taking a mean over the whole set of ensemble members. Instead, recent works have shown that NAJ teleconnections can be used a priori to guide the selection of ensemble members that predict specific dynamical links to sources of high predictability, leading to improved skill [5]. For summer seasonal climate ensemble predictions in NAE, such identification of high skill cases has mainly focused on the Circumglobal Teleconnection Pattern [17]. While this teleconnection is related to the subtropical component of NAJ, predictability associated with the eddy-driven component of NAJ has been less explored in initialised ensemble predictions $[16,18]$.

Going beyond previous work, here we identify forecast opportunities for skillful European summer climate predictions using a neural network approach. We perform the analysis for an EPS based on the Max Planck Institute Earth System Model before assessing the hindcast skill. As a first step, we focus on predictability 
emerging from large-scale atmospheric regimes associated with the combined variability of both components of the NAJ.

\section{METHOD}

\subsection{Indices}

The summer North Atlantic Oscillation (SNAO) and East Atlantic Pattern (EAP) are the two main circulation indices often used as diagnostic tools to quantify changes in speed and location of NAJ $[1,7]$. Together, SNAO and EAP define the dominant summer regimes in NAE. Traditionally, these indices are defined as the first two empirical orthogonal functions of NAE summer sea level pressure (SLP). Whilst SNAO is described by a meridional dipole in SLP between the Icelandic Low and Azores High, EAP has a more zonal dipole structure with pressure centres shifted southeastwards compared to those of the SNAO. However, because EAP seems to modulate the surface expression of SNAO, both indices are necessary to describe NAJ variability [24]. Positive (negative) SNAO (EAP) phases correspond to anomalously northerly position of NAJ. In NAE these phases are associated with warmer and drier conditions in northern and central regions, and colder and wetter over south. Generally, negative (positive) SNAO (EAP) phases show a reverse pattern. As an alternative to this two-valued classification, we use a finer classification of NAE teleconnection patterns [12] to evaluate NAJ variability and to target windows of opportunity in an EPS.

\subsection{Datasets}

2.2.1 Reanalysis. We define our finer classification of teleconnection patterns as the dominant summer large-scale atmospheric regimes occurring throughout the 20th century in the NAE sector $\left(70^{\circ} \mathrm{W}-40^{\circ} \mathrm{E}, 25^{\circ}-80^{\circ} \mathrm{N}\right)$. For this purpose we use July and August monthly means SLP from ERA-20C reanalysis [19] covering the period of 1900-2010. The classification is obtained by the neural network classifier Self-Organising Maps (SOM).

2.2.2 Model output. Our second dataset is a set of 30-member hindcast simulations with the Max Planck Institute Earth System Model in its mixed-resolution setup (MPI-ESM-MR) covering the period 1902-2008. The atmospheric component ECHAM6 [21] has a resolution of T63L95, and the oceanic component MPI-OM [13] has a resolution of TP04L40. External forcing is taken from CMIP5 [8]. We use July and August monthly means (3-4 months lead time) of SLP, geopotential height at $500 \mathrm{hPa}(\mathrm{Z} 500)$ and total precipitation from this hindcast.

An ensemble of 30-member 6-month long hindcasts is initialized in May every year from 1902-2008. Initial conditions for hindcasts are taken from an assimilation experiment. The assimilation experiment is performed using the MPI-ESM-MR with full-field nudging by Newtonian relaxation towards all atmospheric and ocean levels except in the boundary layer. The atmosphere conditions of vorticity, divergence, three-dimensional temperature and two-dimensional pressure are taken from ERA-20C. In the ocean, three-dimensional daily mean salinity and temperature anomalies are nudged at a relaxation time of approximately 10 days. The ocean state is derived in an ocean-only simulation performed with MPI-OM forced with the atmospheric variables from ERA-20C. The three-dimensional atmospheric and ocean fields of the assimilation experiment form the initial conditions, and ensemble members are generated by small perturbations of the atmospheric state by disturbing the diffusion coefficient in the uppermost layer.

2.2.3 Preprocessing. At every gridpoint we compute anomalies by removing seasonal cycle and linear trend, in order to eliminate the centennial-scale climate change signal. We use July-August seasonal means (JA) to focus on the low-frequency dominant summer atmospheric regimes. In addition, all data have been weighted by the cosine of latitude to take into account the dependence of the gridpoint density on latitude. The final dataset used for training consist of 111 gridded SLP maps composed of 30x59 pixels at approximately $1.875^{\circ}$ resolution.

\subsection{Self-Organising Maps}

We use JA SLP fields from ERA-20C reanalysis to train a Python implementation of Self-Organising Map (SOM) [22]. SOM is a nonlinear method based on unsupervised learning with two-layer neural networks [14]. SOM's architecture allows for a reduced and ordered representation of high-dimensional datasets by a smaller set of variables. The SOM algorithm also provides a metric among those latent variables. This finer classification sets the basis for efficient application of SOM to explore large-scale, slow varying processes. Consequently, SOM has been successfully used for climate characterisation (e.g. $[11,20])$.

In a typical two-layer SOM, the input layer corresponds to feature vectors $\mathbf{z}$ from dataset $\mathbf{D}$, while the output layer is the SOM map. The SOM map is a topological ordering of neurons usually in 2D grid (denoted $S O M_{i j}$, where $i$ and $j$ are the grid indices of the SOM map). This layer is fully connected to the input layer via weight vectors $\mathbf{w}$ with the same dimension as $\mathbf{z}$. The lattice structure of the layers permit to calculate a measure of distance (here Euclidean distance) and identify the shortest path between neurons of both layers, thereby assigning as Best Matching Unit the respective closest neuron in the SOM map, iteratively. A fundamental property of SOM is the topological ordering: neighbouring neurons $S O M_{i j}$ represent similar neurons in the input data space and therefore share similar properties. Hereafter we adopt mode as terminology to refer to neurons in the SOM map, i.e. $S O M_{i j}$. For details on the SOM algorithm see [15].

In agreement with previous studies for the region, the classification is obtained on a a $5 \times 5$ rectangular lattice of neurons. We choose training parameters as a compromise to keeping low quantisation and topological errors, while achieving a detailed view on the representative patterns associated with combined $\mathrm{SNAO}(+/-)$ and $\mathrm{EAP}(+/-)$ indices. We find that optimum parameters are $i)$ initial spread of the neighbourhood function $\sigma(0)=0.55$ (Gaussian) and ii) learning rate 0.4 , for a maximum of 10000 iterations in batch training mode.

To facilitate interpretation of the SOM modes as corresponding to one of the four dominant summer regimes (see 2.1), we choose a Hierarchical Agglomerative Clustering algorithm using Ward dissimilarity to objectively cluster similar SOM modes as representative patterns for the dominant summer regimes $(k=4)$. [10]. Note that during learning, each observation in $\mathbf{D}$ is associated with only one mode $S O M_{i j}$ in the trained SOM map. In other words, this 
analysis assigns one dominant cluster per summer each year, i.e. a time series of the observed dominant summer regimes $\left(C_{o b s}\right)$.

\subsection{Ensemble subsampling}

We perform an ensemble subsampling with the aim of leveraging the MPI-EPS before calculating the ensemble mean and assessing the hindcast skill. This procedure assumes that retaining only ensemble members which predict the observed dominant summer regime $\left(C_{o b s}\right)$ will improve the forecast skill in MPI-EPS. We refer to this refinement as observed-mode strategy. We perform the following steps, per hindcast year (1902 - 2008):

(1) project JA SLP mean of each $n$ ensemble member $Y_{n y}$ onto the SOM map

(2) calculate RMSE between each pairs $\left(Y_{n y}, S O M_{i j}\right)$

(3) denote winner the $S O M_{i j}$ for which RMSE is minimum (i.e. most similar mode)

(4) verify to which cluster the winner mode belongs, i.e. $C_{\text {win }}$

(5) retain $Y_{n y}$ in the ensemble only if $C_{o b s}=C_{\text {win }}$.

This procedure selects a subsample of potential skillful ensemble members per year. This selection of ensemble members is used to calculate the ensemble mean of SLP, Z500 and TP.

\subsection{Seasonal hindcast skill analysis}

The hindcast skill of MPI-EPS against the ERA-20C data is assessed using two skill scores. The first is point-wise detrended anomaly correlation coefficient (ACC) [4], which describes the model ability to reproduce the reference anomalies and have values in the range $[-1,1]$ : the higher the score, the better. The second skill metric is the spread-error ratio [9], which measures the EPS reliability in representing the full uncertainty range as realistic as possible. Thus, the mean of the ensemble spread should be about equal to the RMSE over the same period (i.e. ideally equal one). An EPS is overdispersed (underdispersed) if the spread-error ratio is greater (smaller) than one, leading to unreliable forecasts if uncalibrated.

\section{EVALUATION}

We present results for a preliminary hindcast analysis with a set of 10 ensemble members. We first evaluate the model ability to predict the observed dominant summer regimes $C_{y}$. We find that regimes associated with a northerly position of NAJ and warmer/drier conditions in northern Europe (SNAO+ and EAP-) are more frequent from 70s onward (Fig. 1 top). This is in line with previous studies which suggest a poleward shift of NAJ in response to anthropogenic forcing (e.g. [16, 23]). Our results suggest that potential predictability in the model shows a marked variability, with about a third of MPI-EPS members predicting the observed dominant summer regime per year (Fig. 1 bottom). The model seems to strive the most in predicting years dominated by EAP, likely reflecting a poor representation of Circumglobal Teleconnection Pattern as in e.g. [2].

In Fig. 2 we show results of seasonal hindcast skill analysis of 3-4 months lead time SLP anomalies for the $1902-2008$ period, focusing in a section of Europe $\left(35^{\circ} \mathrm{N}-70^{\circ} \mathrm{N}, 10^{\circ} \mathrm{W}-30^{\circ} \mathrm{E}\right)$. Analysis for Z500 show overall similar results (not shown). We compare skill metrics for the full ensemble and the observed-mode strategy with selected members. With the full ensemble, we find very limited
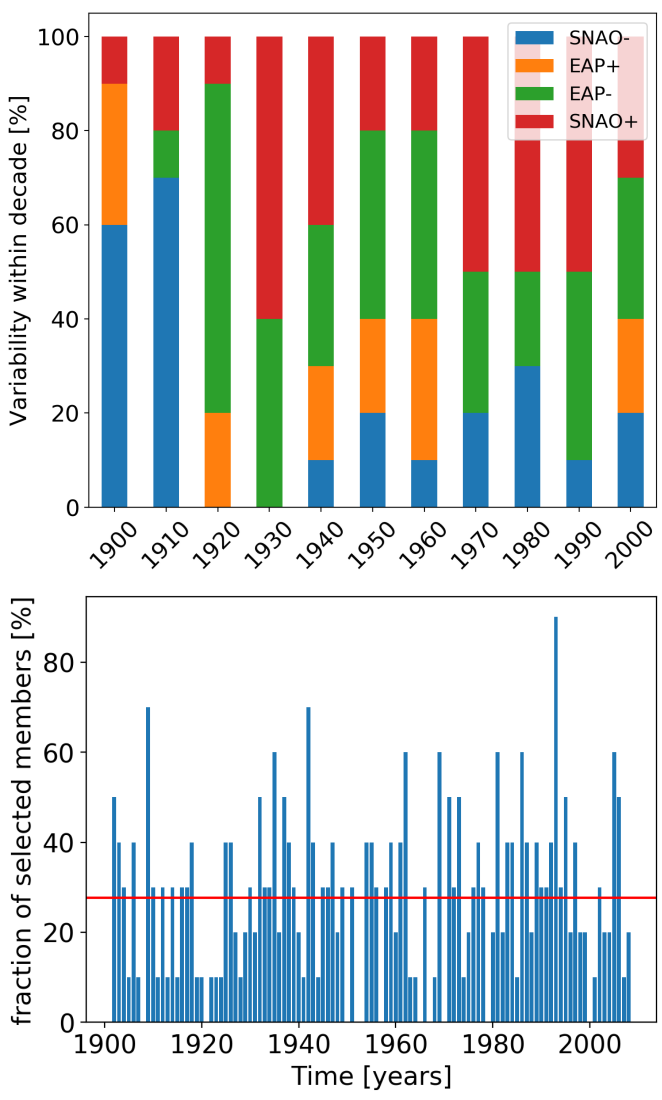

Figure 1: Top: Low-frequency variability of the dominant clusters of summer regimes within each decade. For example, the first bar denotes years 1900-1909 and shows the percentage of years within this decade belonging to each cluster (colour coded). Bottom: Fraction of MPI-ESM-MR ensemble members that predict the observed dominant clusters of summer regimes.

predictive skill over Europe (Fig. 2 top-left), about $1 \%$ of agreement with ERA-20C (Fig. 2 top-right). One reason for the limited skill is the high overdispersion of ensemble members in most Europe (Fig. 2 bottom-left). Assuming that this overdispersion reflects the influence of different physical processes related to NAJ variability, we now target predictions with the observed-mode strategy to select ensemble members. We first compare the ensemble mean calculated with the full and selected members (Fig. 3 bottom). A consequence of the subsampling is the higher temporal variability of the selected ensemble mean, since the ensemble spread is reduced for every start date. We achieve better agreement with ERA-20C, as illustrated by the linear regression line of selected members being much closer to the line of perfect linear regression (grey line). We find significantly higher skill for JA SLP anomalies, particularly over the northern sector (Fig. 2 bottom), and improved spread-errorratios of about 1 in many areas (Fig. 2 bottom-right). The mean ACC increases to about $40 \%$ agreement with ERA-20C (Fig. 3 top). Highest ACC improvement is found over regions of marked SNAO 
influence. Lower improvement in the southern sector is probably due to influence of teleconnections not targeted in our analysis, such as Indian Monsoon (e.g. [6]). Our results are in line with a previous analysis of this set of simulations for Z500 using k-means clustering [16].
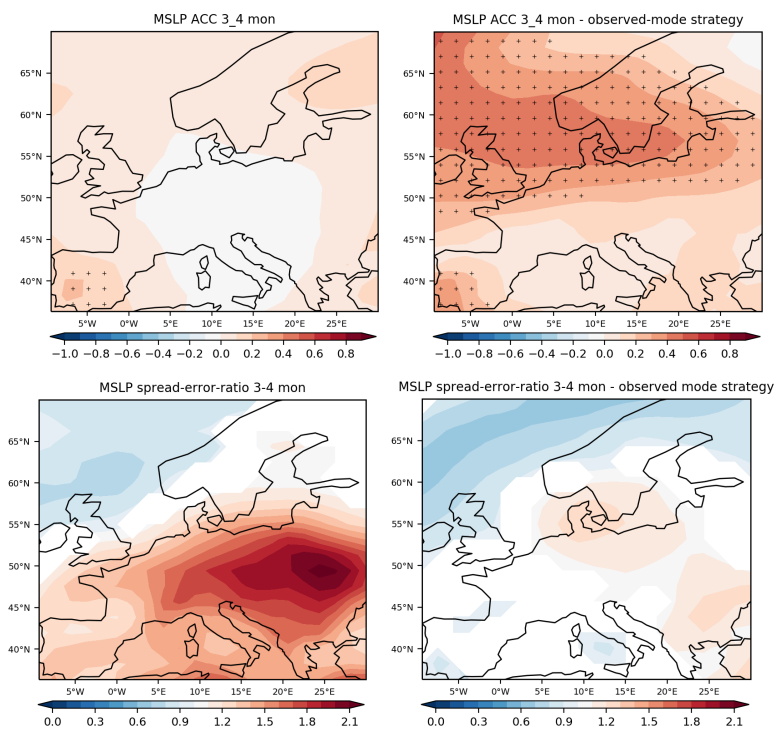

Figure 2: MPI-ESM-MR skill against ERA-20C for 3-4 mon lead time SLP anomalies for the 1902-2008 period. (top) ACC considering the full ensemble (left) and the selected ensemble for the European region (right). Stippling represents significance at the $95 \%$ confidence level. (bottom) Spread-error ratios for the full (left) and selected ensemble (right). White shading corresponds to ratio $=\mathbf{1} \pm \mathbf{0 . 0 5}$.

\section{CONCLUSIONS \& FUTURE WORK}

We show a methodology that combines dynamical ensemble predictions and selection of ensemble members by Self-Organising Maps to systematically find forecast opportunities for European summer climate skillful forecasts. By targeting the correct representation of the two dominant modes of summertime North Atlantic Jet variability in the ensemble prediction system, we better evaluate the model performance. Our subsampling approach leverages the EPS ensemble mean, achieving improved skill scores for May predictions of July-August SLP and Z500.

Our ongoing analyses build on recent studies that link the largescale atmospheric regimes investigated here with tropical [17] and subtropical [18] spring sea surface temperatures in the North Atlantic. We currently perform a flow-dependent hindcast skill verification based on spring sea surface temperature patterns preceding these atmospheric summer regimes. This analysis is crucial to verify the potential of performing an ensemble selection before initialisation.
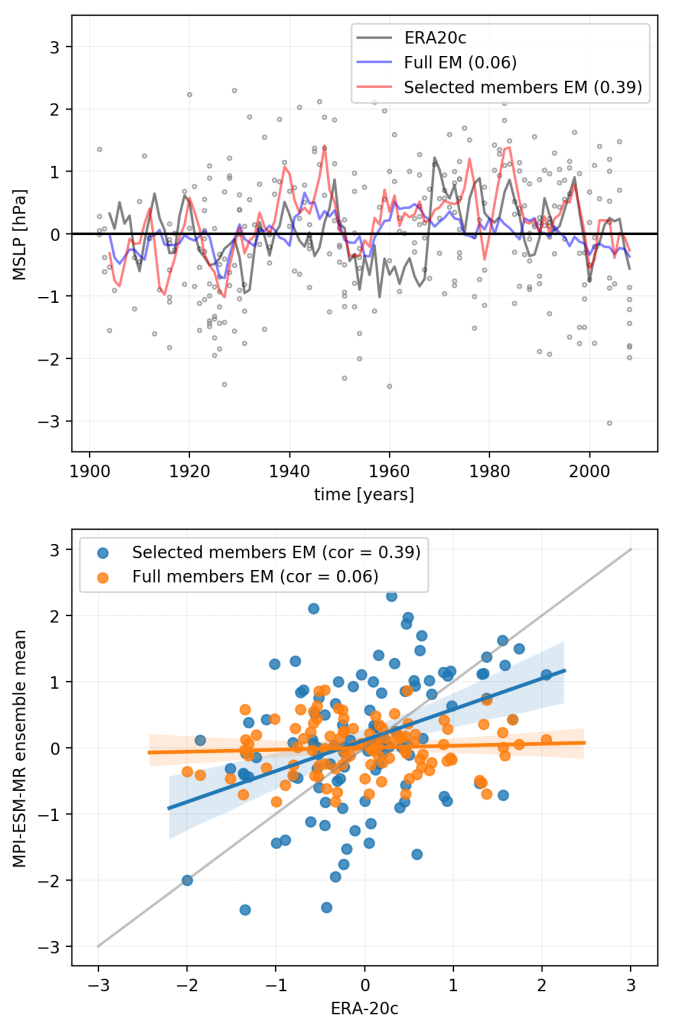

Figure 3: (top) Comparison between ERA20C (grey line) and the ensemble mean over the full ensemble (blue line) and the selected ensemble (red line) from MPI-ESM in 1902-2008 for the region $35^{\circ} \mathrm{N}-70^{\circ} \mathrm{N}, 10^{\circ} \mathrm{W}-30^{\circ} \mathrm{E}$. Grey circles indicate the value of each ensemble member. Time series are smoothed with a 3-month running mean. (bottom) Scatterplots of ERA20C compared to the ensemble mean of MPIESM in 1902-2008 over the full (orange circles) or the selected ensemble (blue circles) including linear regression.

\section{ACKNOWLEDGMENTS}

This study is also part of the pilot-project 'Reduced Complexity Models' (Redmod) of the Incubator Data Science Initiative of the Helmholtz Association in Germany.

\section{REFERENCES}

[1] Ana Bastos, Ivan A Janssens, Célia M Gouveia, Ricardo M Trigo, Philippe Ciais, Frédéric Chevallier, Josep Penuelas, Christian Rödenbeck, Shilong Piao, Pierre Friedlingstein, et al. 2016. European land CO 2 sink influenced by NAO and East-Atlantic Pattern coupling. Nature communications 7, 1 (2016), 1-9.

[2] Jonathan D Beverley, Steven J Woolnough, Laura H Baker, Stephanie J Johnson, and Antje Weisheimer. 2019. The northern hemisphere circumglobal teleconnection in a seasonal forecast model and its relationship to European summer forecast skill. Climate dynamics 52, 5-6 (2019), 3759-3771.

[3] Ileana Bladé, Brant Liebmann, Didac Fortuny, and Geert Jan van Oldenborgh. 2012. Observed and simulated impacts of the summer NAO in Europe: implications for projected drying in the Mediterranean region. Climate dynamics 39, 3-4 (2012), 709-727.

[4] Matthew Collins. 2002. Climate predictability on interannual to decadal time scales: The initial value problem. Climate Dynamics 19, 8 (2002), 671-692.

[5] Mikhail Dobrynin, Daniela IV Domeisen, Wolfgang A Müller, Louisa Bell, Sebastian Brune, Felix Bunzel, André Düsterhus, Kristina Fröhlich, Holger Pohlmann, 
and Johanna Baehr. 2018. Improved teleconnection-based dynamical seasonal predictions of boreal winter. Geophysical Research Letters 45, 8 (2018), 3605-3614.

[6] Buwen Dong, Rowan T Sutton, Tim Woollings, and Kevin Hodges. 2013. Variability of the North Atlantic summer storm track: mechanisms and impacts on European climate. Environmental Research Letters 8, 3 (2013), 034037.

[7] Chris K Folland, Jeff Knight, Hans W Linderholm, David Fereday, Sarah Ineson, and James W Hurrell. 2009. The summer North Atlantic Oscillation: past, present, and future. Journal of Climate 22, 5 (2009), 1082-1103.

[8] Marco A Giorgetta, Johann Jungclaus, Christian H Reick, Stephanie Legutke, Jürgen Bader, Michael Böttinger, Victor Brovkin, Traute Crueger, Monika Esch, Kerstin Fieg, et al. 2013. Climate and carbon cycle changes from 1850 to 2100 in MPI-ESM simulations for the Coupled Model Intercomparison Project phase 5. fournal of Advances in Modeling Earth Systems 5, 3 (2013), 572-597.

[9] Chun Kit Ho, Ed Hawkins, Len Shaffrey, Jochen Bröcker, Leon Hermanson, James M Murphy, Doug M Smith, and Rosie Eade. 2013. Examining reliability of seasonal to decadal sea surface temperature forecasts: The role of ensemble dispersion. Geophysical Research Letters 40, 21 (2013), 5770-5775.

[10] Anil K Jain and Richard C Dubes. 1988. Algorithms for clustering data. PrenticeHall, Inc.

[11] Nathaniel C Johnson. 2013. How many ENSO flavors can we distinguish? Journal of Climate 26, 13 (2013), 4816-4827.

[12] Nathaniel C Johnson, Steven B Feldstein, and Bruno Tremblay. 2008. The continuum of Northern Hemisphere teleconnection patterns and a description of the NAO shift with the use of self-organizing maps. Journal of Climate 21, 23 (2008), 6354-6371.

[13] JH Jungclaus, Nils Fischer, Helmuth Haak, K Lohmann, J Marotzke, D Matei, U Mikolajewicz, D Notz, and JS Von Storch. 2013. Characteristics of the ocean simulations in the Max Planck Institute Ocean Model (MPIOM) the ocean component of the MPI-Earth system model. Fournal of Advances in Modeling Earth Systems 5, 2 (2013), 422-446.

[14] Teuvo Kohonen. 1984. Self-Organization and Associative Memory. Springer.
[15] Teuvo Kohonen. 2013. Essentials of the self-organizing map. Neural networks 37 (2013), 52-65.

[16] Nele Neddermann. 2019. Seasonal prediction of European summer climate: a process-based approach. Ph.D. Dissertation. Universität Hamburg Hamburg.

[17] Nele-Charlotte Neddermann, Wolfgang A Müller, Mikhail Dobrynin, André Düsterhus, and Johanna Baehr. 2018. Seasonal predictability of European summer climate re-assessed. Climate Dynamics (2018), 1-18.

[18] Albert Ossó, Rowan Sutton, Len Shaffrey, and Buwen Dong. 2020. Development, Amplification, and Decay of Atlantic/European Summer Weather Patterns Linked to Spring North Atlantic Sea Surface Temperatures. Fournal of Climate 33, 14 (2020), 5939-5951.

[19] Paul Poli, Hans Hersbach, Dick P Dee, Paul Berrisford, Adrian J Simmons, Frédéric Vitart, Patrick Laloyaux, David GH Tan, Carole Peubey, Jean-Noël Thépaut, et al. 2016. ERA-20C: An atmospheric reanalysis of the twentieth century. Fournal of Climate 29, 11 (2016), 4083-4097.

[20] Irene Polo, Albin Ullmann, Pascal Roucou, and Bernard Fontaine. 2011. Weather regimes in the Euro-Atlantic and Mediterranean sector, and relationship with West African rainfall over the 1989-2008 period from a self-organizing maps approach. Journal of Climate 24, 13 (2011), 3423-3432.

[21] Bjorn Stevens, Marco Giorgetta, Monika Esch, Thorsten Mauritsen, Traute Crueger, Sebastian Rast, Marc Salzmann, Hauke Schmidt, Jürgen Bader, Karoline Block, et al. 2013. Atmospheric component of the MPI-M Earth system model: ECHAM6. Fournal of Advances in Modeling Earth Systems 5, 2 (2013), 146-172.

[22] Giuseppe Vettigli. 2019. MiniSom: minimalistic and NumPybased implementation of the Self Organizing Map. Release 2.1.5. http://github.com/JustGlowing/ minisom.

[23] Tim Woollings and Mike Blackburn. 2012. The North Atlantic jet stream under climate change and its relation to the NAO and EA patterns. fournal of Climate 25, 3 (2012), 886-902.

[24] Tim Woollings, Abdel Hannachi, and Brian Hoskins. 2010. Variability of the North Atlantic eddy-driven jet stream. Quarterly fournal of the Royal Meteorological Society 136, 649 (2010), 856-868. 